\title{
Definition of the upper reference limit for thyroglobulin antibodies according to the National Academy of Clinical Biochemistry guidelines: comparison of eleven different automated methods
}

\author{
F. D'Aurizio ${ }^{1}$ - P. Metus $^{2}$ - A. Ferrari ${ }^{3}$ - B. Caruso ${ }^{3}$ - R. Castello ${ }^{4}$.

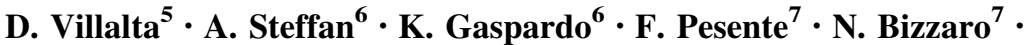

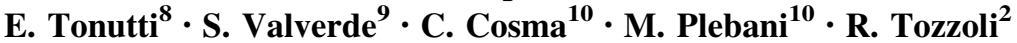 \\ Received: 30 April 2017 / Accepted: 6 June 2017/Published online: 19 June 2017 \\ (c) The Author(s) 2017. This article is an open access publication
}

\begin{abstract}
Purpose In the last two decades, thyroglobulin autoantibodies ( $\mathrm{Tg} \mathrm{Ab})$ measurement has progressively switched from marker of thyroid autoimmunity to test associated with thyroglobulin $(\mathrm{Tg})$ to verify the presence or absence of $\mathrm{TgAb}$ interference in the follow-up of patients with differentiated thyroid cancer. Of note, $\mathrm{TgAb}$ measurement is cumbersome: despite standardization against the International Reference Preparation MRC 65/93, several studies demonstrated high inter-method variability and wide variation in limits of detection and in reference intervals.
\end{abstract}

F. D'Aurizio

federica.daurizio@asuiud.sanita.fvg.it

1 Clinical Pathology Institute, University Hospital, P.le S. Maria della Misericordia, 33100 Udine, Italy

2 Clinical Pathology Laboratory, 'S. Maria degli Angeli' Hospital, Pordenone, Italy

3 Clinical Pathology Laboratory, University Hospital, Verona, Italy

4 General Medicine and Endocrinology, University Hospital, Verona, Italy

5 Allergology and Immunology, 'S. Maria degli Angeli' Hospital, Pordenone, Italy

6 Oncological Clinical Pathology Laboratory, CRO, IRCCS, Aviano, Italy

7 Clinical Pathology Laboratory, 'S. Antonio Hospital', Tolmezzo, Italy

8 Laboratory of Immunopathology and Allergology, University Hospital, Udine, Italy

9 Laboratory Medicine, 'Madonna della Navicella' Hospital, Chioggia (Ve), Italy

10 Department of Laboratory Medicine, University Hospital, Padua, Italy
Taking into account the above considerations, the main aim of the present study was the determination of $\mathrm{TgAb}$ upper reference limit (URL), according to the National Academy of Clinical Biochemistry guidelines, through the comparison of eleven commercial automated immunoassay platforms.

Methods The sera of 120 healthy males, selected from a population survey in the province of Verona, Italy, were tested for $\mathrm{TgAb}$ concentration using eleven IMA applied on as many automated analyzers: AIA-2000 (AIA) and AIA-CL2400 (CL2), Tosoh Bioscience; Architect (ARC), Abbott Diagnostics; Advia Centaur XP (CEN) and Immulite 2000 XPi (IMM), Siemens Healthineers; Cobas 6000 (COB), Roche Diagnostics; Kryptor (KRY), Thermo Fisher Scientific BRAHMS, Liaison XL (LIA), Diasorin; Lumipulse G (LUM), Fujirebio; Maglumi 2000 Plus (MAG), Snibe and Phadia 250 (PHA), Phadia AB, Thermo Fisher Scientific. All assays were performed according to manufacturers' instructions in six different laboratories in Friuli-Venezia Giulia and Veneto regions of Italy [Lab 1 (AIA), Lab 2 (CL2), Lab 3 (ARC, COB and LUM), Lab 4 (CEN, IMM, KRY and MAG), Lab 5 (LIA) and Lab 6 (PHA)]. Since $\mathrm{TgAb}$ values were not normally distributed, the experimental URL (e-URL) was established at 97.5 percentile according to the non-parametric method.

Results TgAb e-URLs showed a significant inter-method variability. Considering the same method, e-URL was much lower than that suggested by manufacturers (mURL), except for ARC and MAG. Correlation and linear regression were unsatisfactory. Consequently, the agreement between methods was poor, with significant bias in Bland-Altman plot.

Conclusions Despite the efforts for harmonization, $\mathrm{TgAb}$ methods cannot be used interchangeably. Therefore, additional effort is required to improve analytical performance 
taking into consideration approved protocols and guidelines. Moreover, TgAb URL should be used with caution in the management of differentiated thyroid carcinoma patients since the presence and/or the degree of $\mathrm{TgAb}$ interference in $\mathrm{Tg}$ measurement has not yet been well defined.

Keywords Autoimmune thyroid disease - Thyroglobulin autoantibodies - Upper reference limit · Immunoassay . Harmonization

$\begin{array}{ll}\text { Abbreviations } \\ \text { 2G } & \text { Second generation } \\ \text { AIA } & \text { AIA-2000, Tosoh Bioscience } \\ \text { ARC } & \text { Architect, Abbott Diagnostics } \\ \text { CEN } & \text { Advia Centaur XP, Siemens Healthineers } \\ \text { CL2 } & \text { AIA-CL2400 (CL2), Tosoh Bioscience } \\ \text { CLSI } & \text { Clinical and Laboratory Standards Institute } \\ \text { COB } & \text { Cobas 6000, Roche Diagnostics } \\ \text { DTC } & \text { Differentiated thyroid carcinoma } \\ \text { FS } & \text { Functional sensitivity } \\ \text { IMA } & \text { Immunometric assay } \\ \text { IMM } & \text { Immulite 2000 XPi, Siemens Healthineers } \\ \text { KRY } & \text { Kryptor, Thermo Fisher Scientific BRAHMS } \\ \text { LIA } & \text { Liaison XL, Diasorin } \\ \text { LOD } & \text { Limit of detection } \\ \text { LUM } & \text { Lumipulse G, Fujirebio } \\ \text { MAG } & \text { Maglumi 2000 Plus, Snibe } \\ \text { NACB } & \text { National Academy of Clinical Biochemistry } \\ \text { PHA } & \text { Phadia 250, Phadia AB, Thermo Fisher } \\ & \text { Scientific } \\ \text { Tg } & \text { Thyroglobulin } \\ \text { TgAb } & \text { Thyroglobulin autoantibodies } \\ \text { TPOAb } & \text { Thyroid peroxidase antibodies } \\ \text { URL } & \text { Upper reference limit } \\ & \end{array}$

\section{Introduction}

Human thyroglobulin (Tg) is a high molecular weight $(660 \mathrm{kDa})$ soluble glycoprotein, typically stored within the follicular colloid of the thyroid, acting as the substrate for thyroid hormones (triiodothyronine, T3 and thyroxine, T4). As $\mathrm{Tg}$ is produced and utilized entirely by benign or differentiated malignant thyroid cells, it is considered a good tumor marker for patients with differentiated thyroid carcinoma (DTC) $[1,2]$ after removal of benign and malignant thyroid tissue by surgery and $\mathrm{I}^{131}$ ablation. Over the years, advances in assay technologies have led to important improvements in the analytical performances of $\mathrm{Tg}$ immunometric assays (IMAs); above all, the functional sensitivity (FS) of Tg IMAs has greatly improved: from 0.5 to $1.0 \mu \mathrm{g} / \mathrm{L}$ of the first generation IMAs to $0.05-0.10 \mu \mathrm{g} / \mathrm{L}$ of the second generation (2G) IMAs [3].

Nevertheless, the major limitation of 2G IMA testing is interference by serum $\mathrm{Tg}$ autoantibodies $(\mathrm{Tg} \mathrm{Ab})$ causing, as a rule, underestimation of $\mathrm{Tg}$ results and possibly masking disease recurrence [4-6]: it has been hypothesized that the complex between free $\mathrm{Tg}$ and endogenous $\mathrm{TgAb}$ prevents free $\mathrm{Tg}$ from binding to the capture and/or signal monoclonal antibody reagents and/or alternatively, endogenous $\mathrm{TgAb}$ binding to free $\mathrm{Tg}$ masks the epitopes recognized by monoclonal antibody reagents $[5,7]$.

Serum $\mathrm{TgAb}$ are reported to be present in about $25-30 \%$ of DTC patients depending of the assay used and the cutoff employed to classify samples as positive or negative $[1,7]$. They are more frequent in females [8] and they are also present in about $60 \%$ of patients with autoimmune thyroid disease (AITD) [9]. On the basis of these considerations, the role of $\mathrm{TgAb}$ measurement has evolved from a marker of thyroid autoimmunity $[10,11]$ to a test associated with $\mathrm{Tg}$ to investigate $\mathrm{TgAb}$ interference [12]. Consequently, serum $\mathrm{TgAb}$ have evolved as a surrogate test for tumor marker replacing $\mathrm{Tg}$ determination by IMAs, in cases of analytical interference from $\operatorname{TgAb}[13,14]$.

Of note, the measurement of $\mathrm{TgAb}$ could be cumbersome. Analytical limitations of serum $\operatorname{TgAb}$ assays have been reported in the context of thyroid autoimmunity diagnosis [9]. Despite standardization against the International Reference Preparation (IRP) MRC 65/93, several studies demonstrated a high variability in the analytical performances of different TgAb IMAs: large variation in limits of detection (LOD), FS, inter-method results, reference intervals with poor concordance between $\mathrm{TgAb}$ assays in patients with DTC [15-22]. The difficulty in standardization is in part due to the heterogeneous $\mathrm{Tg}$ immunoreactivity: differential splicing of Tg mRNA, various posttranslational modifications, and alterations of biosynthesis regulation in thyroid tumor cells lead to exposure or masking of epitopes with resulting differences in $\mathrm{Tg}$ immunologic structure [23]. Besides $\mathrm{Tg}$ heterogeneity, assay discordance has also been assigned to various specificity of circulating $\mathrm{TgAb}$ in patient sera [6]. As a result, different $\operatorname{TgAb}$ values are obtained when the same serum is tested with different methods [15-22]. Finally, differences in assay reagents, above all the preparation of the antigen $(\mathrm{Tg})$, definitely contribute to assay variability [1-12].

The manufacturers' upper reference limit (URL) for $\mathrm{TgAb}$, set up to identify patients with AITD but misleading for evaluation of $\mathrm{TgAb}$ interference in $\mathrm{Tg}$ assay, is another aspect to consider. Reference intervals are the most widely used tool for the interpretation of clinical laboratory results. The Clinical and Laboratory Standards Institute (CLSI) Expert Panel on Reference Values has provided 
guidelines for the determination of reliable reference intervals (EP28-A3c) [24]. They recommended the use of the direct method, which implies the enrolment of a healthy population of at least 120 individuals and the determination of 2.5th and 97.5th percentile for the lower reference limit and the URL, respectively. As regards thyroid antibodies (thyroid peroxidase antibodies-TPOAb and $\mathrm{TgAb}$ ) for AITD diagnosis, the 2003 proposal of the National Academy of Clinical Biochemistry (NACB) recommends the use of a direct method and a reference group composed of
120 men younger than 30 years, biochemically euthyroid [i.e., with serum thyrotropin stimulating hormone (TSH), concentrations between 0.5 and $2.0 \mathrm{mIU} / \mathrm{L}]$, and without risk parameters (goiter, family history of AITD, or other autoimmune diseases) [25].

However, the definition of the TgAb URL remains a matter of debate, because of the problems in enrolling the appropriate reference group [25] and in the determination of $\mathrm{TgAb}$ cut-off suitable for the identification of assay

Table 1 Analytical performance characteristics of the current TgAb automated immunoassays

\begin{tabular}{|c|c|c|c|c|c|c|c|}
\hline Method & $\begin{array}{l}\text { Immunoassay } \\
\text { principle }\end{array}$ & Tracer/enzyme & $\begin{array}{l}\text { Assay } \\
\text { type }\end{array}$ & $\begin{array}{l}\text { Imprecision }(\%) \text { : } \\
\text { intra-; inter-; total }\end{array}$ & $\begin{array}{l}\mathrm{LoD}^{\mathrm{d}} \\
(\mathrm{IU} / \mathrm{mL})\end{array}$ & $\begin{array}{l}\mathrm{LoQ}^{\mathrm{d}} \\
(\mathrm{IU} / \mathrm{mL})\end{array}$ & $\begin{array}{l}\text { Assay range } \\
(\mathrm{IU} / \mathrm{mL})\end{array}$ \\
\hline AIA & FEIA & $\begin{array}{l}\text { 4MUP/ } \\
\text { Alkaline phosphatase }\end{array}$ & $\mathrm{NC}$ & $\begin{array}{l}\text { 4.3-5.1; nd; } \\
5.5-6.0\end{array}$ & 0.12 & nd & $0.12-2000$ \\
\hline ARC & CLIA & Acridinium esters & $\mathrm{NC}$ & $\begin{array}{l}1.7-6.6^{\mathrm{b}} ; \mathrm{nd} ; \\
2.7-8.2^{\mathrm{b}}\end{array}$ & 0.07 & 0.31 & $0.07-1000$ \\
\hline $\mathrm{CEN}^{\mathrm{a}}$ & CLIA & Acridinium esters & $\mathrm{C}$ & $\begin{array}{l}2.9-5.5 ; 1.8-2.0 \\
3.5-5.8\end{array}$ & 10 & $30^{\mathrm{e}}$ & $10-500$ \\
\hline CL2 & CLEIA & Difurat $^{\circledR}$ & $\mathrm{NC}$ & $\begin{array}{l}5.1-5.5 ; \\
5.8-6.6 ; \text { nd }\end{array}$ & 0.005 & nd & $0.005-2500$ \\
\hline $\mathrm{COB}$ & ECLIA & Ruthenium derivatives & $\mathrm{C}$ & $\begin{array}{l}1.3-5.6^{\mathrm{c}} \\
2.1-8.7^{\mathrm{c}} ; \text { nd }\end{array}$ & 10 & nd & $10-4000$ \\
\hline IMM & CLIA & $\begin{array}{l}\text { Adamantyl dioxetane } \\
\text { phosphate/Alkaline } \\
\text { phosphatase }\end{array}$ & $\mathrm{NC}$ & $\begin{array}{l}3.2-4.9 ; \\
4.6-5.8 ; \text { nd }\end{array}$ & 2.2 & nd & $20-3000$ \\
\hline$K R Y^{\mathrm{a}}$ & TRACE & $\begin{array}{l}\text { Europium cryptate/ } \\
\text { XL } 665\end{array}$ & $\mathrm{C}$ & $\begin{array}{l}1.5-3.5 ; \\
6.8-20.0 ; \text { nd }\end{array}$ & 10 & 33 & $10-850$ \\
\hline LIA & CLIA & Isoluminol derivatives & $\mathrm{NC}$ & $\begin{array}{l}2.3-3.2 ; \\
4.4-8.9 ; \text { nd }\end{array}$ & 5 & 10 & $5-5000$ \\
\hline LUM & CLEIA & AMPPD & $\mathrm{NC}$ & $\begin{array}{l}1.8-4.6 ; \mathrm{nd} \\
2.5-5.3^{\mathrm{c}}\end{array}$ & 5.152 & 5.152 & $5.152-3000$ \\
\hline MAG & CLIA & ABEI & $\mathrm{NC}$ & $\begin{array}{l}2.8-9.1 ; \\
5.2-9.8 ; \text { nd }\end{array}$ & 10 & nd & $10-2800$ \\
\hline PHA & FIA & $\begin{array}{l}\text { 4-methyl-umbellipheryl- } \beta \text {-D- } \\
\text { galactoside/ } \beta \text {-galactosidase }\end{array}$ & $\mathrm{NC}$ & $\begin{array}{l}3.3-5.6 ; \\
2.6-6.5 ; \text { nd }\end{array}$ & 12 & nd & $12-4794$ \\
\hline
\end{tabular}

$4 M U P$ 4-methyl-umbelliferyl phosphate, ABEI $N$-(aminobutil)- $N$-(ethyl)-isoluminol, $A I A$ AIA-2000, Tosoh Bioscience, $A M P P D$ alkaline phosphatase-spiroadamantyl-methoxy-phosphoryloxy-phenyl-dioxetane, $A R C$ Architect, Abbott Diagnostics, $C$ competitive immunoassay, $C E N$ Advia Centaur XP, Siemens Healthineers, CL2 AIA CL-2400, Tosoh Bioscience, CLIA chemiluminescence immunoassay, CLEIA chemiluminescence enzyme immunoassay, COB Cobas 6000, Roche Diagnostics, ECLIA electrochemiluminescence immunoassay, FEIA fluorescence enzyme immunoassay, FIA fluoroimmunoassay, IMM Immulite $2000 \mathrm{XPi}$, Siemens Healthineers, KRY Kryptor, Thermo Fisher Scientific BRAHMS, LIA Liaison XL, Diasorin, LUM Lumipulse G, Fujirebio, MAG Maglumi 2000 Plus, Snibe, NC non-competitive immunoassay, nd not declared, PHA Phadia 250, Phadia AB, Thermo Fisher Scientific, TRACE time resolved amplified cryptate emission

a All methods are standardized with the reference preparation MRC 65/93 and use International Units (IU/mL) except for Centaur and Kryptor which refer to a secondary standard and use Arbitrary Units (AU/mL); to obtain IU multiply for the conversion factor 2.8 (CEN) and 7.14 (KRY)

${ }^{b}$ Precision defined by the NCCLS Protocol EP5-A [26]

${ }^{c}$ Precision defined by the modified NCCLS Protocol EP5-A2 [27]

${ }^{\mathrm{d}}$ LoD and LoQ defined by the CLSI protocol EP17-A [28]

e Functional sensitivity defined as TgAb concentration with total $\mathrm{CV} \leq 20 \%$, determined for a period of two days using one lot of reagents and testing, by four instruments, multiple samples from normal patients 
Fig. 1 Distribution of $\mathrm{TgAb}$ values for each method. AIA AIA-2000, Tosoh Bioscience, ARC Architect, Abbott Diagnostics, CEN Advia Centaur XP, Siemens Healthineers, $C I$ confidence intervals, CL2 AIA CL-2400, Tosoh Bioscience, $C O B$ Cobas 6000, Roche Diagnostics, IMM Immulite $2000 \mathrm{XPi}$, Siemens Healthineers, $K R Y$ Kryptor, Thermo Fisher Scientific BRAHMS, LIA Liaison XL, Diasorin, LUM Lumipulse G, Fujirebio, MAG Maglumi 2000 Plus, Snibe, No. number, $P H A$ Phadia 250, Phadia AB, Thermo Fisher Scientific, $R S D$ relative standard deviation. $S D$ standard deviation

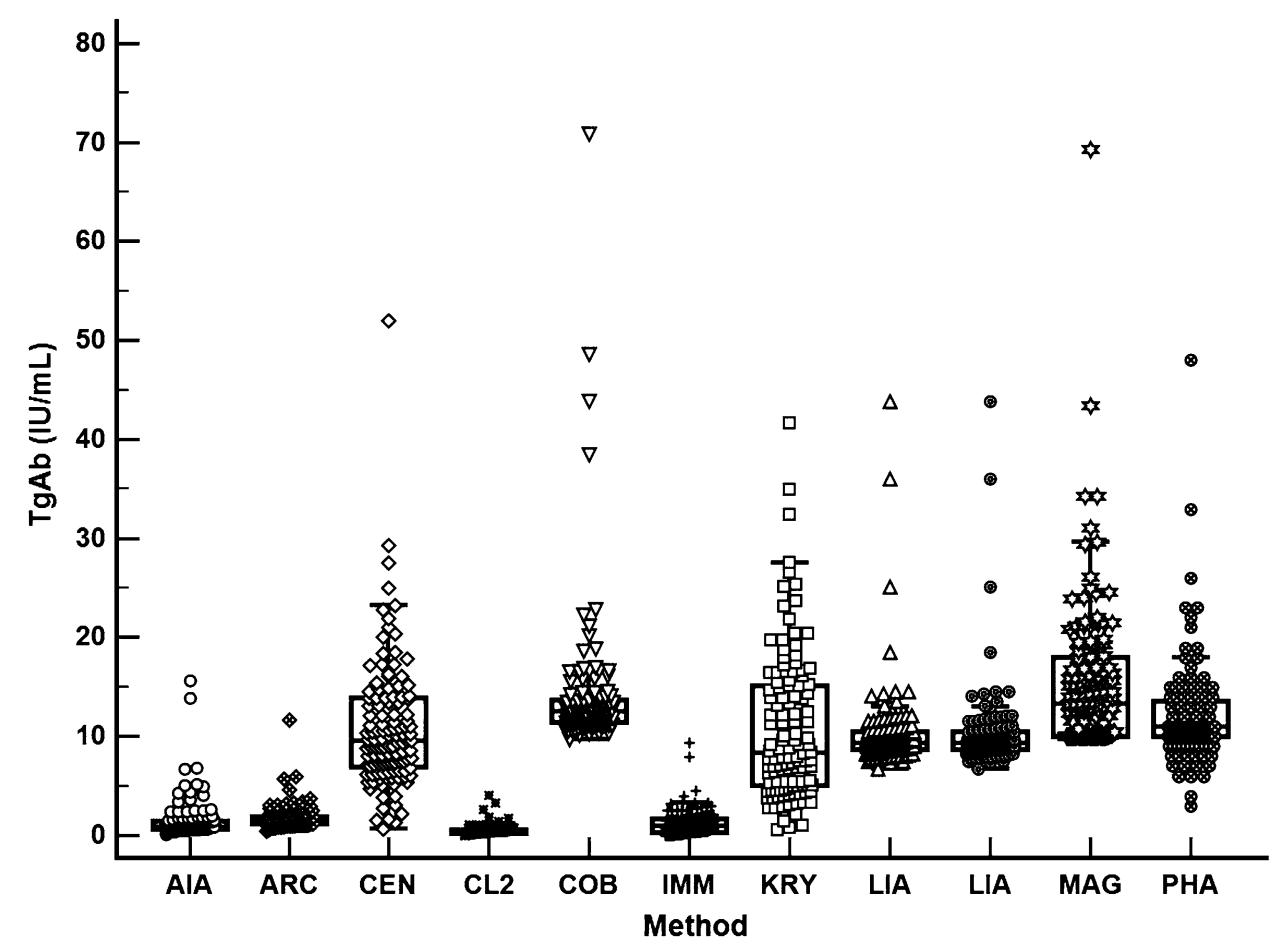

interference and consequently for the use of $\mathrm{TgAb}$ as surrogate marker in the follow-up of DTC [12].

Taking into account the above considerations, the main aim of the present study was the determination of $\mathrm{TgAb}$ URL, according to the NACB guidelines, by the use of eleven commercial automated IMA platforms. A further aim of the study was to compare the analytical performances of the methods used, in an attempt to evaluate, whenever possible, their effectiveness in detecting $\mathrm{TgAb}$ interference.

\section{Materials and methods}

One hundred and twenty male subjects were selected from a population survey in the province of Verona, Italy, according to the NACB criteria [25]. All of them gave informed consent for their participation in the study. Their sera were tested for $\operatorname{TgAb}$ concentration by using eleven IMA methods applied in as many automated analyzers: AIA-2000 (AIA) and AIA-CL2400 (CL2), Tosoh Bioscience; Architect (ARC), Abbott Diagnostics; Advia Centaur XP (CEN) and Immulite 2000 XPi (IMM), Siemens Healthineers; Cobas 6000 (COB), Roche Diagnostics; Kryptor (KRY), Thermo Fisher Scientific BRAHMS, Liaison XL (LIA), Diasorin; Lumipulse G (LUM), Fujirebio; Maglumi 2000 Plus (MAG), Snibe and Phadia 250 (PHA), Phadia AB, Thermo Fisher Scientific. All assays were performed according to manufacturers' instructions at six different laboratories in Friuli-Venezia
Giulia and Veneto regions of Italy [Lab 1 (AIA), Lab 2 (CL2), Lab 3 (ARC, COB and LUM), Lab 4 (CEN, IMM, KRY and MAG), Lab 5 (LIA) and Lab 6 (PHA)]. The main features of the eleven methods are summarized in Table 1 . All methods are standardized with the reference preparation (IRP MRC 65/93) and use International Units (IU), except for CEN and KRY whose results were initially expressed in Arbitrary Units and then converted in IU (Table 1). The normality of the distribution was assessed using the Shapiro-Wilk test. Since $\operatorname{TgAb}$ values were not normally distributed, the experimental URL (e-URL) was established at 97.5th according to the non-parametric percentile method (CLSI standard C28-A3c) [24]. Moreover, the non-parametric Kruskal-Wallis test and the Dunn's multiple comparison test were used for comparing the median values of the eleven groups.

The inter-method variability was assessed considering the interquartile range (25th and 75th percentile). To compare the eleven methods, ARC was regarded as the reference assay since it showed a satisfactory combination between the LoD and the assay imprecision (Table 1). Correlation between assays was assessed by Spearman Rank correlation coefficient $\left(r_{\mathrm{s}}\right)$; Passing-Bablok regression was applied to verify the linear association between methods, while agreement between assays was analyzed by Bland-Altman plot considering the difference between ARC and the other ten methods (AIA, CEN, CL2, COB, IMM, KRY, LIA, LUM, MAG and PHA). The difference between manufacturer's URL (m-URL) and e-URL was expressed as the ratio between them in percentage 


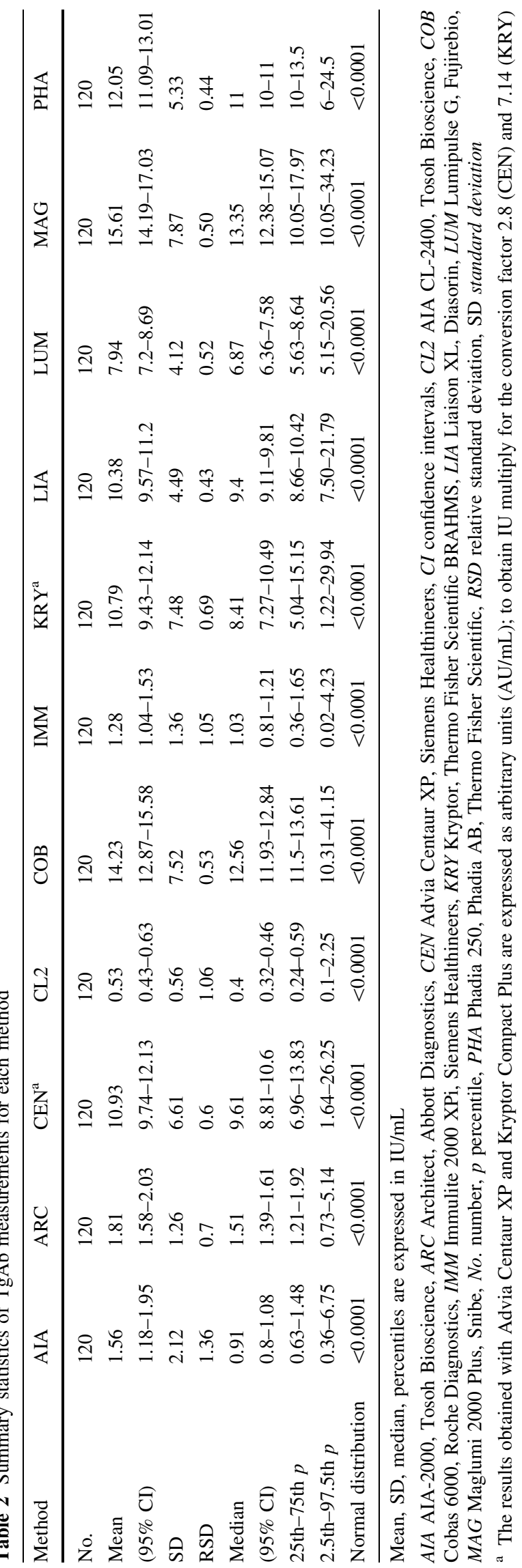

$($ Delta $\%=\mid \mathrm{m}-\mathrm{URL}-\mathrm{e}-\mathrm{URL} / \mathrm{m}-\mathrm{URL} \times 100)$. A twosided value of $p<0.05$ was considered statistically significant. Statistical analyses were performed by GraphPad Prism Software, version 4.0 (San Diego, CA, USA) and MedCalc software, version 11.6 (Ostend, Belgium).

\section{Results}

$\mathrm{TgAb}$ results showed a relevant inter-method variability with wide interquartile ranges: the difference reached 48 times for the 25th percentile (minimum: $0.24 \mathrm{IU} / \mathrm{mL}$ and maximum: $11.5 \mathrm{IU} / \mathrm{mL}$ ) and 30 times for 75 th percentile (minimum: $0.59 \mathrm{IU} / \mathrm{mL}$, maximum: $17.97 \mathrm{IU} / \mathrm{mL}$ ) (Fig. 1) (Table 2).

A statistically significant difference between medians was observed for all methods except for 11 pairs of the 45 combinations analyzed (Fig. 1) (Table 3).

e-URLs differed from one method to the other. Of note, within the same method, e-URL was much lower than m-URL, except for ARC and MAG, which showed similar values for both (Table 4).

As regards the correlations between methods, $r_{\mathrm{s}}$ ranged from 0.17 (ARC vs CEN) to 0.56 (ARC vs CL2) (Table 5). Using Passing-Bablok analysis, $\mathrm{TgAb}$ method comparison resulted in varying degrees of agreement with the reference method (ARC). Slopes were all far from 1 except for ARC vs AIA (slope $=1.15$ ) and ARC vs CL2 (0.34) (Fig. 2) (Table 5); intercepts varied from -29.92 to 3.7, they were far from 0 except for ARC vs AIA (-0.75) and ARC vs CL2 (-0.15) (Fig. 2) (Table 5). Subsequently, a relevant positive or negative mean biases were observed by BlandAltman analysis ranging from $-115.8 \%$ (CL2 vs ARC) to $156.4 \%$ (MAG vs ARC). The best agreement was between AIA and ARC with a mean bias of $-37 \%$ (Fig. 3) (Table 6).

\section{Discussion}

The determination of the cut-off for the definition of $\mathrm{TgAb}$ positivity is an important and controversial issue.

In this study, we have determined the TgAb URL in a reference group of male individuals, meticulously defined as being free of thyroid diseases, by eleven IMA methods, currently used in autoimmunology laboratories, and compared to each other. Actually, to our knowledge, no similar data are present in literature: in the past, other studies faced the same topic but with small numbers of different analytical methods, most of which are no longer in use [9, 15-22, 29].

The first relevant result of the present study was the demonstration of differences between TgAb URLs claimed 
Table 3 Kruskal-Wallis test and Dunn's multiple comparison test of $\mathrm{TgAb}$ methods: comparison of all pairs of columns

\begin{tabular}{ll}
\hline A. Kruskal-Wallis test & \\
\hline No. of groups & 11 \\
$p$ Value & $p<0.0001$ \\
Do the medians vary significantly $(p<0.05)$ & Yes \\
\hline B. Dunn's multiple comparison test & $p$ value \\
\hline AIA vs ARC & $p>0.05$ \\
AIA vs IMM & $p>0.05$ \\
ARC vs IMM & $p>0.05$ \\
CEN vs KRY & $p>0.05$ \\
CEN vs LIA & $p>0.05$ \\
CEN vs LUM & $p>0.05$ \\
CL2 vs IMM & $p>0.05$ \\
COB vs MAG & $p>0.05$ \\
KRY vs LIA & $p>0.05$ \\
KRY vs LUM & $p>0.05$ \\
LIA vs LUM & $p>0.05$
\end{tabular}

A There is a significant difference between the medians of the eleven groups. B In the table are reported only the comparison of groups with non-significant differences

AIA AIA-2000, Tosoh Bioscience, ARC Architect, Abbott Diagnostics, CEN Advia Centaur XP, Siemens Healthineers, $C I$ confidence intervals, CL2 AIA CL-2400, Tosoh Bioscience, COB Cobas 6000, Roche Diagnostics, IMM Immulite $2000 \mathrm{XPi}$, Siemens Healthineers, $K R Y$ Kryptor, Thermo Fisher Scientific BRAHMS, LIA Liaison XL, Diasorin, LUM, Lumipulse G, Fujirebio, MAG, Maglumi 2000 Plus, Snibe, No. number, PHA Phadia 250, Phadia AB, Thermo Fisher Scientific in the package insert (m-URL) and those obtained in the male reference sample (e-URL): with the exception of ARC and MAG method, e-URLs were lower than those proposed by the manufacturers, the difference ranging from 2.33 to $88.85 \%$. These results were similar to those described in two previous studies dealing with the definition of TPOAb reference limits, determined by several current IMA platforms [30, 31]. In our opinion, these discrepancies could be related to the lack of strict criteria in the selection of the subjects for the reference group. Specifically, racial differences could play some role, as most of the studies, sponsored by manufacturers, were performed in the geographical area of the production line and consequently difficult to reproduce in other settings. Moreover, the use of non-stringent criteria in the choice of subjects could have led to the enrolment of individuals with subclinical AITD, thus resulting in relatively high levels of $\mathrm{TgAb}$ causing the raise of the 97.5th percentile of the reference value distribution platforms [32-37].

The second relevant consideration that emerged from the present study was the variation of e-URLs according to the method used. The e-URL ranged from 2.25 (CL2) to $41.15 \mathrm{IU} / \mathrm{mL}$ (COB), with an approximately 18 -fold variation, consistent with a previous paper which reported the same magnitude of variation using five IMA methods distinct from those considered in the present study (18). The difference between e-URLs supports concerns regarding inter-method variation [38]. Specifically, there were relevant differences between methods in terms of
Table 4 Experimental upper reference limit compared to the manufacturer's upper reference limit for most of the current $\mathrm{TgAb}$ automated immunoassays, established from a cohort of 120 euthyroid control subjects

\begin{tabular}{lllll}
\hline Method & No. & m-URL $(\mathrm{IU} / \mathrm{mL})$ & e-URL $($ C.I. $90 \%)(\mathrm{IU} / \mathrm{mL})$ & Delta $(\%)$ \\
\hline AIA & 120 & 13.6 & $6.82(5-15.7)$ & 49.85 \\
ARC & 120 & 4.11 & $5.66(3.29-11.64)$ & 37.71 \\
CEN $^{\mathrm{a}}$ & 120 & 60 & $27.44(21.9-52)$ & 54.27 \\
CL2 & 120 & 6.8 & $2.63(1.15-4.08)$ & 61.32 \\
COB & 120 & 115 & $43.69(21.16-70.88)$ & 62 \\
IMM & 120 & 40 & $4.46(3.26-9.41)$ & 88.85 \\
KRY & 120 & 33 & $32.23(25.14-41.61)$ & 2.33 \\
LIA & 120 & 100 & $24.93(14.37-43.91)$ & 75.07 \\
LUM & 120 & 55.4 & $21.07(12.3-32.8)$ & 61.97 \\
MAG & 120 & 30 & $34.23(29.46-69.36)$ & 14.1 \\
PHA & 120 & 60 & $25.93(21-48)$ & 56.78 \\
\hline
\end{tabular}

e-URL: 97.5th percentile; Delta $=$ Im-URL - e-URLI/m-URL $\times 100$

AIA AIA-2000, Tosoh Bioscience, ARC Architect, Abbott Diagnostics, CEN Advia Centaur XP, Siemens Healthineers, $C I$ confidence intervals, $C L 2$ AIA CL-2400, Tosoh Bioscience, COB Cobas 6000, Roche Diagnostics, $e-U R L$ experimental upper reference limit, IMM Immulite $2000 \mathrm{XPi}$, Siemens Healthineers, $K R Y$ Kryptor, Thermo Fisher Scientific BRAHMS, LIA Liaison XL, Diasorin, LUM Lumipulse G, Fujirebio, $m$-URL manufacturer upper reference limit, MAG Maglumi 2000 Plus, Snibe, No. number, PHA Phadia 250, Phadia AB, Thermo Fisher Scientific

${ }^{a}$ The results obtained with Advia Centaur XP and Kryptor Compact Plus are expressed as arbitrary units (AU/mL); to obtain IU multiply for the conversion factor 2.8 (CEN) and 7.14 (KRY) 


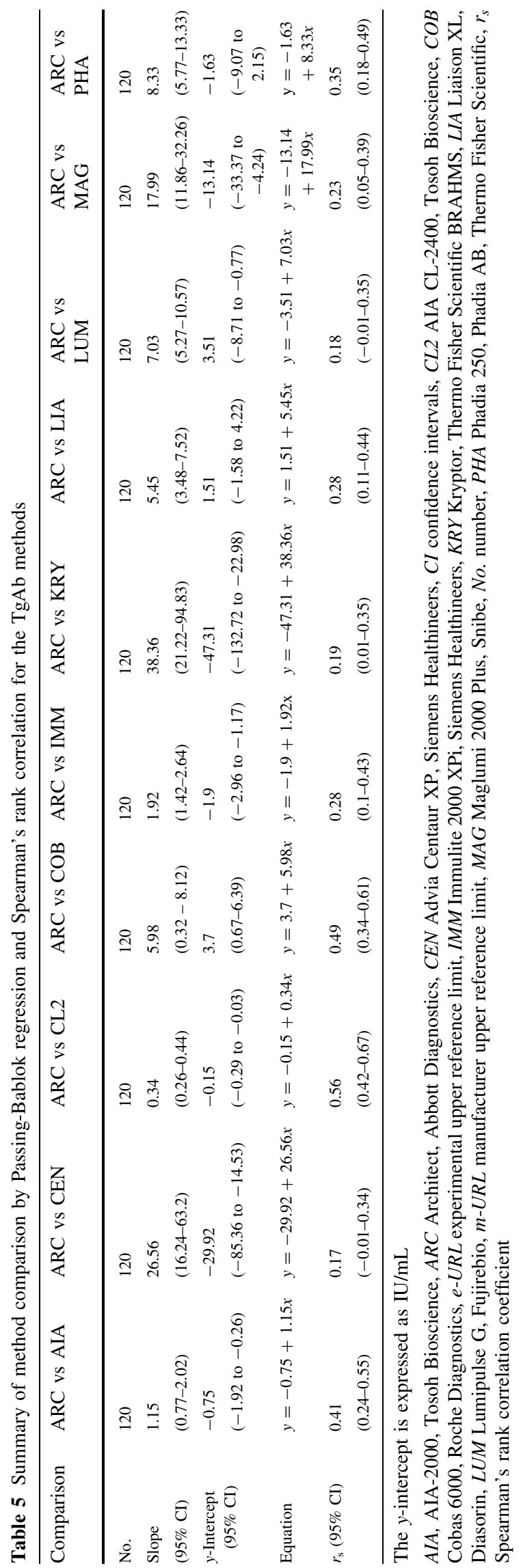

medians (31-fold) $(p<0.05$, Kruskal-Wallis test) and interquartile ranges. These discrepancies were not expected and not easily explained; in fact, in recent decades, there have been significant improvements in harmonization between methods [39], resulting from the high level of automation of analytical procedures and the use of the same reference preparation (IRP MRC 65/93). Moreover, analytical imprecision seems not contribute to the above differences, as the values declared by the individual manufacturer were essentially overlapping (although obtained with different protocols, some of them standardized, some others not) and in general lower than $10 \%$ for both intraand inter-assay imprecision (Table 1). Such discordance between $\operatorname{Tg} \mathrm{Ab}$ assays could be attributed to various factors, including: (1) TgAb heterogeneity which is often independent to standardization efforts, and which implies different specificity for Tg antigen; (2) Tg interference and (3) differences in assay reagents, including solid phase material and the preparations of the antigen ( $\mathrm{Tg}$ ), which could affect the proper exposure of the immunodominant epitopes. Another important aspect to consider, to explain inter-method variability, was the diverse assay structures of the eleven IMA methods leading to a different LoD (Table 1) ranging from 0.005 to $12 \mathrm{IU} / \mathrm{mL}$. Especially, a clear-cut discrepancy between methods with a LoD lower than $0.2 \mathrm{IU} / \mathrm{mL}$ (ARC, AIA and CL2) and methods with a LoD equal to or higher than $2 \mathrm{IU} / \mathrm{mL}$ was apparent.

To better evaluate the relationship between methods, ARC was chosen as the reference method on the basis of the best combination between LoD and imprecision (Table 1): the correlation of ARC with the other methods was not satisfactory, in line with the variability of the results, broadly described above. Passing-Bablok regression did not show a satisfactory agreement between assays. Furthermore, consistent with regression results, BlandAltman plot highlighted a statistically significant positive or negative mean biases.

The lack of acceptable agreement between methods has relevant practical implications: clinicians have to use the same method to monitor $\mathrm{TgAb}$ concentration in the followup of DTC, on the other hand, laboratories must keep users timely informed about any modification in $\mathrm{TgAb}$ method to simplify re-baselining.

Despite the analysis of the data showed satisfactory analytical performances of some methods in terms of LoD, being able to measure also low levels of $\mathrm{TgAb}$ with adequate precision, the main limitation to this study lay in having contributed only indirectly to the debated question of $\mathrm{TgAb}$ analytical interference. In fact, the obtained results did not prove but only suggested the opportunity to choose the more sensitive and accurate latest generation methods for measuring $\mathrm{TgAb}$, to better detect any false negative results even in patients with $\mathrm{TgAb}$ levels lower 


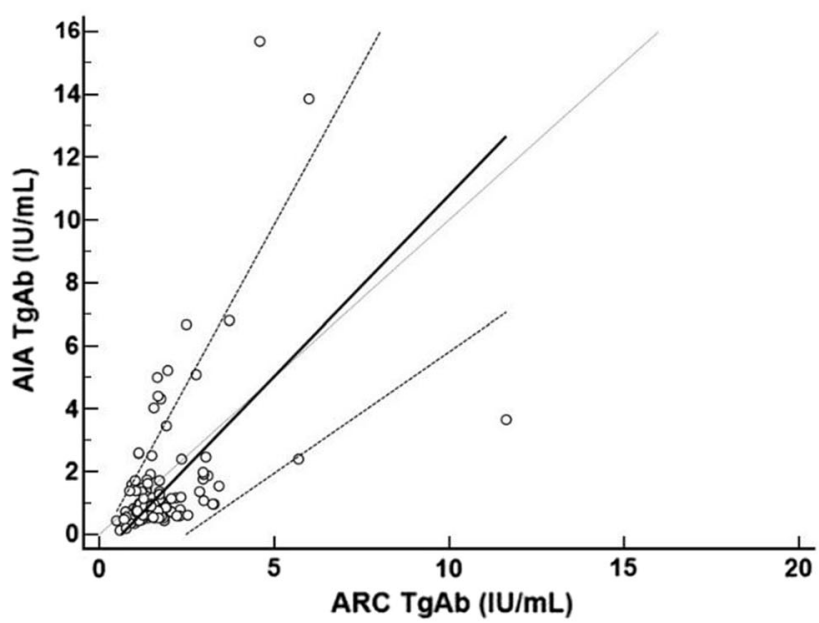

Fig. 2 Passing-Bablok regression of $\mathrm{TgAb}$ methods. ARC was chosen as the reference method on the $x$ axis. ARC vs AIA and ARC vs CL2 showed the best relationship in terms of slope and

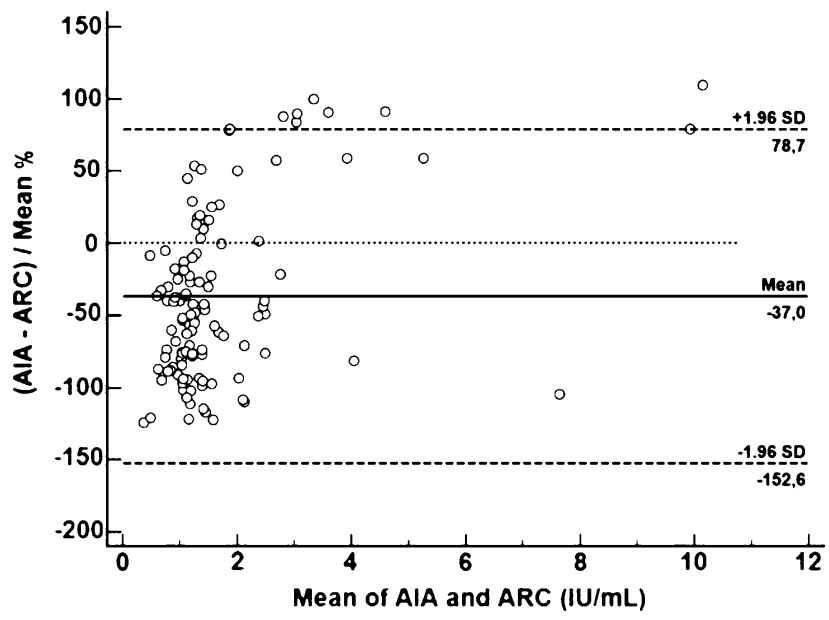

Fig. 3 Bland-Altman plots showing the difference between ARC and AIA and between ARC and CL2. ARC was chosen as the reference method. An ideal mean difference of 0 is indicated by a dotted line, the mean difference by a solid line and the limits of agreement for the

than the cut-off (the so-called "negative patient"). Therefore, according to these considerations, two different cutoffs for $\mathrm{TgAb}$ could be proposed, one for the diagnosis of AITD and one for the effects of $\mathrm{TgAb}$ on $\mathrm{Tg}$ measurement.

\section{Conclusions}

In spite of the attempt of harmonization, quantitative agreement between methods was generally not satisfactory and methods could not be used interchangeably.

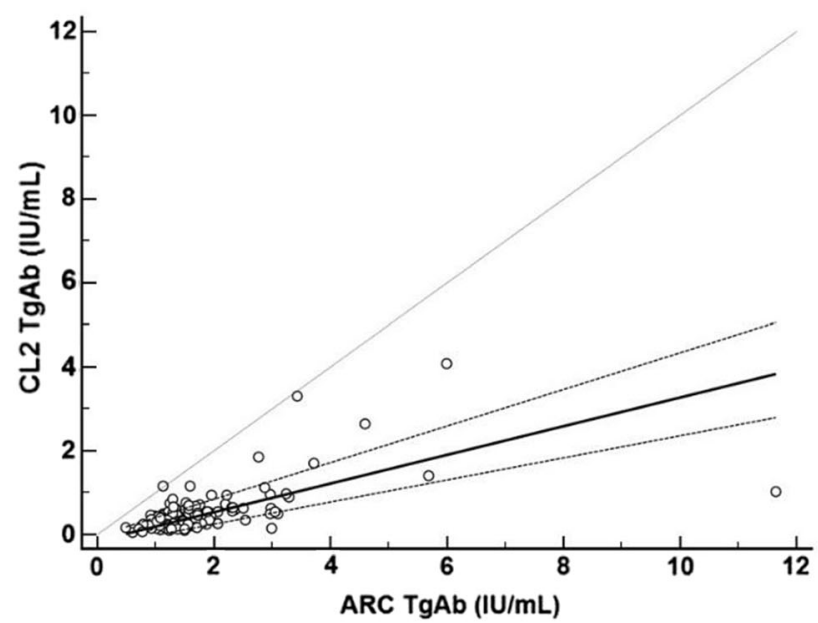

intercept. AIA AIA-2000, Tosoh Bioscience, ARC Architect, Abbott Diagnostics, CL2 AIA CL-2400, Tosoh Bioscience

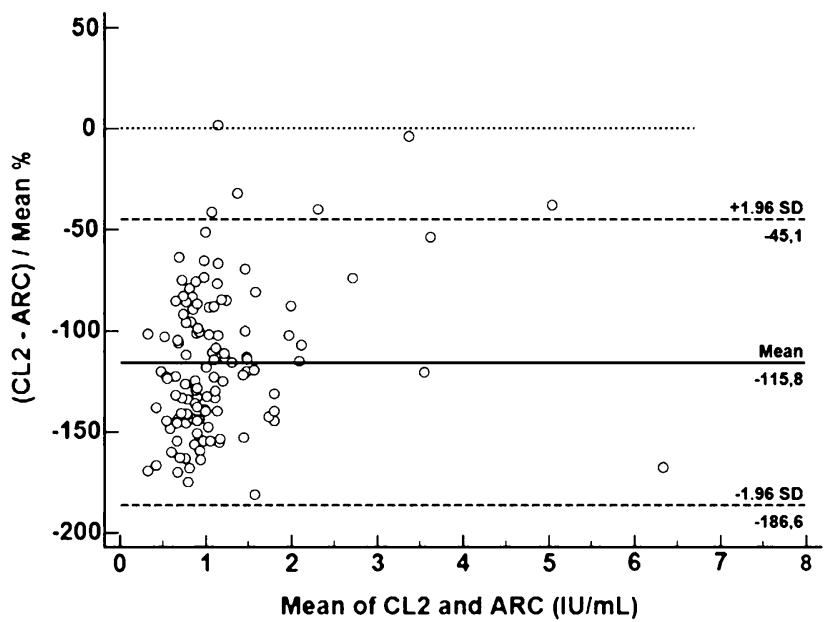

mean difference, as defined by $95 \%$ confidence limits, by dashed lines. AIA AIA-2000, Tosoh Bioscience, ARC Architect, Abbott Diagnostics, CL2 AIA CL-2400, Tosoh Bioscience

Therefore, additional standardization efforts are required to improve analytical performance, and biomedical industries are strongly invited to re-evaluate their assays taking into consideration CLSI approved protocols and guidelines.

Finally, as long as the relationship between $\mathrm{TgAb}$ concentration and interference in $\mathrm{Tg}$ measurement is not clearly defined, $\operatorname{TgAb}$ URL must be used with caution, taking into account that it is usually set for the diagnosis of AITD and not for the identification of potential interference in Tg assay. 
Acknowledgements We thank all participant centres for their excellent technical support and the industry's manufacturers for providing reagents.

\section{Compliance with ethical standards}

Ethical approval The Thyroid Study, conducted in the province of Verona, was approved by the local ethics committee.

Informed consent Informed consent was obtained from all the participants in the study.

Conflict of interest The authors declare that they have no conflict of interest.

Open Access This article is distributed under the terms of the Creative Commons Attribution 4.0 International License (http://crea tivecommons.org/licenses/by/4.0/), which permits unrestricted use, distribution, and reproduction in any medium, provided you give appropriate credit to the original author(s) and the source, provide a link to the Creative Commons license, and indicate if changes were made.

\section{References}

1. Evans C, Tennant S, Perros P (2015) Thyroglobulin in differentiated thyroid cancer. Clin Chim Acta 444:310-317

2. Grebe SK (2009) Diagnosis and management of thyroid carcinoma: focus on serum thyroglobulin. Expert Rev Endocrinol Metab 4:25-43

3. Spencer C, Petrovic I, Fatemi S, LoPresti J (2014) Serum thyroglobulin $(\mathrm{Tg})$ monitoring of patients with differentiated thyroid cancer using sensitive (second-generation) immunometric assays can be disrupted by false-negative and false-positive serum thyroglobulin autoantibody misclassifications. J Clin Endocrinol Metab 99:4589-4599

4. Giovanella L, Feldt-Rasmussen U, Verburg FA et al (2015) Thyroglobulin measurement by highly sensitive assays: focus on laboratory challenges. Clin Chem Lab Med 53:1301-1314

5. Locsei Z, Szabolcs I, Rácz K et al (2012) Serum thyroglobulin antibody levels within or near to the reference range may interfere with thyroglobulin measurement. Biochem Med 22:365-370

6. Spencer C, Petrovic I, Fatemi S (2011) Current thyroglobulin autoantibody (TgAb) assays often fail to detect interfering $\mathrm{TgAb}$ that can result in the reporting of falsely low/undetectable serum Tg IMA values for patients with differentiated thyroid cancer. J Clin Endocrinol Metab 96:1283-1291

7. Görges R, Maniecki M, Jentzen W et al (2005) Development and clinical impact of thyroglobulin antibodies in patients with differentiated thyroid carcinoma during the first 3 years after thyroidectomy. Eur J Endocrinol 153:49-55

8. Spencer CA, Takeuchi M, Kazarosyan M et al (1998) Serum thyroglobulin autoantibodies: prevalence, influence on serum thyroglobulin measurement, and prognostic significance in patients with differentiated thyroid carcinoma. J Clin Endocrinol Metab 83:1121-1127

9. Tozzoli R, Bizzaro N, Tonutti E et al (2002) Italian Society of Laboratory Medicine (SIMeL) Study Group on the Laboratory Diagnosis of Autoimmune Diseases. Immunoassay of anti-thyroid autoantibodies: high analytical variability in second generation methods. Clin Chem Lab Med 40:568-573

10. Hollowell JG, Staehling NW, Flanders WD et al (2002) Serum TSH, T(4), and thyroid antibodies in the United States population 
(1988-1994): National Health and Nutrition Examination Survey (NHANES III). J Clin Endocrinol Metab 87:489-499

11. Latrofa F, Ricci D, Montanelli L et al (2012) Lymphocytic thyroiditis on histology correlates with serum thyroglobulin autoantibodies in patients with papillary thyroid carcinoma: impact on detection of serum thyroglobulin. J Clin Endocrinol Metab 97:2380-2387

12. Spencer C, Fatemi S (2013) Thyroglobulin antibody (TgAb) methods-Strengths, pitfalls and clinical utility for monitoring TgAb-positive patients with differentiated thyroid cancer. Best Pract Res Clin Endocrinol Metab 27:701-712

13. Chiovato L, Latrofa F, Braverman LE et al (2003) Disappearance of humoral thyroid autoimmunity after complete removal of thyroid antigens. Ann Intern Med 139:346-351

14. Verburg FA, Luster M, Cupini C et al (2013) Implications of thyroglobulin antibody positivity in patients with differentiated thyroid cancer: a clinical position statement. Thyroid 23:12111225

15. Bohuslavizki KH, vom Baur E E, Weger B et al (2000) Evaluation of chemiluminescence immunoassays for detecting thyroglobulin (Tg) and thyroid peroxidase (TPO) autoantibodies using the IMMULITE 2000 system. Clin Lab 46:23-31

16. Gilmour J, Brownlee Y, Foster P et al (2000) The quantitative measurement of autoantibodies to thyroglobulin and thyroid peroxidase by automated microparticle based immunoassays in Hashimoto's disease, Graves' disease and a follow-up study on postpartum thyroid disease. Clin Lab 46:57-61

17. Sapin R, d'Herbomez M, Gasser F et al (2003) Increased sensitivity of a new assay for anti-thyroglobulin antibody detection in patients with autoimmune thyroid disease. Clin Biochem 36:611616

18. La'ulu SL, SLev PR, Roberts WL (2007) Performance characteristics of 5 automated thyroglobulin and thyroperoxidase autoantibody assays. Clin Chim Acta 376:88-95

19. Krahn J, Dembinski $T$ (2009) Thyroglobulin and anti-thyroglobulin assays in thyroid cancer monitoring. Clin Biochem 42:416-419

20. Taylor KP, Parkington D, Bradbury S et al (2011) Concordance between thyroglobulin antibody assays. Ann Clin Biochem 48: 367-369

21. Pickett AJ, Jones M, Evans C (2012) Causes of discordance between thyroglobulin antibody assays. Ann Clin Biochem 49: 463-467

22. Lindberg B, Svensson J, Ericsson UB et al (2001) Comparison of some different methods for analysis of thyroid autoantibodies: importance of thyroglobulin autoantibodies. Thyroid 11:265-269

23. Schulz R, Bethäuser H, Stempka L et al (1989) Evidence for immunological differences between circulating and thyroidderived thyroglobulin in men. Eur J Clin Invest 19:459-463

24. CLSI (2008) Defining, establishing and verifying reference intervals in the clinical laboratory; Approved guideline, 3rd edn, vol 28, No. 30, CLSI document, Wayne

25. Baloch Z, Carayon P, Comte-Devolx B et al (2003) Laboratory medicine practice guidelines. Laboratory support for the diagnosis and monitoring of thyroid disease. Thyroid 13:3-126
26. NCCLS (1999) Evaluation of precision performance of clinical chemistry devices; approved guideline, vol 19, No. 2. NCCLS document EP5-A, Wayne

27. NCCLS (2004) Evaluation of Precision Performance of Quantitative measurement methods; Approved Guideline, 2nd edn, vol 24, No. 25. NCCLS document EP5-A2, Wayne

28. NCCLS (2004) Protocols for determination of limits of detection and limits of quantitation: approved guideline, 2nd edn, vol 24, No. 34. NCCLS publication EP 17-A, NCCLS, Wayne

29. Spencer CA, Bergoglio LM, Kazarosyan M et al (2005) Clinical impact of thyroglobulin $(\mathrm{Tg})$ and $\mathrm{Tg}$ autoantibody method differences on the management of patients with differentiated thyroid carcinomas. J Clin Endocrinol Metab 90:5566-5575

30. Tozzoli R, D'Aurizio F, Ferrari A et al (2016) The upper reference limit for thyroid peroxidase autoantibodies is method-dependent: a collaborative study with biomedical industries. Clin Chim Acta 452:61-65

31. D'Aurizio F, Metus P, Polizzi Anselmo A et al (2015) Establishment of the upper reference limit for thyroid peroxidase autoantibodies according to the guidelines proposed by the National Academy of Clinical Biochemistry: comparison of five different automated methods. Autoimmun Highlights 6:31-37

32. Tozzoli R, Giavarina D, Villalta D et al (2008) Definition of reference limits for autoantibodies to thyroid peroxidase and thyroglobulin in a large population of outpatients using an indirect method based on current data. Arch Pathol Lab Med 132:1924-1928

33. Jensen EA, Petersen PH, Blaabjerg O et al (2006) Establishment of reference distributions and decision values for thyroid antibodies against thyroid peroxidase (TPOAb), thyroglobulin $(\mathrm{Tg} \mathrm{Ab})$ and the thyrotropin receptor (TRAb). Clin Chem Lab Med 44:991-998

34. Kaloumenoum I, Mastorakos G, Alevizaki M et al (2008) Thyroid autoimmunity in schoolchildren in an area with long-standing iodine sufficiency: correlation with gender, pubertal stage and maternal thyroid autoimmunità. Thyroid 18:747-754

35. Taubner K, Schubert G, Pulzer F et al (2014) Serum concentrations of anti-thyroid peroxidase and anti-thyroglobulin antibodies in children and adolescents without apparent thyroid disorders. Clin Biochem 47:3-7

36. Feldt-Rasmussen U, Hoier-Madsen M, Bech K et al (1991) Antithyroid peroxidase antibodies in thyroid disorders and non-thyroid autoimmune diseases. Autoimmunity 9:245-254

37. Mariotti S, Chiovato L, Franceschi C, Pinchera A (1998) Thyroid autoimmunity and aging. Exp Gerontol 33:535-541

38. Wood WG, Hanke R (2004) Comparability of method results and performance in a national external quality assessment scheme between 1993 and 2003 using thyroid associated antibodies as examples. Clin Lab 50:209-221

39. D'Aurizio F, Tozzoli R, Villalta D et al (2015) Immunoassay of thyroid peroxidase autoantibodies: diagnostic performance in automated third generation methods. Clin Chem Lab Med 53: $415-421$ 